\title{
Electron-impact ionization cross section of formic acid ${ }^{\star}$
}

\author{
Mateusz Zawadzki ${ }^{1,2, \text { a }}$ \\ 1 J. Heyrovský Institute of Physical Chemistry, Czech Academy of Sciences, Dolejškova 3, 18223 Prague, \\ Czech Republic \\ 2 Atomic Physics Division, Department of Atomic, Molecular, and Optical Physics, Faculty of Applied Physics and \\ Mathematics, Gdańsk University of Technology, ul. G. Narutowicza 11/12, 80-233 Gdańsk, Poland
}

Received 20 August 2017 / Received in final form 3 November 2017

Published online 18 January 2018

(C) The Author(s) 2018. This article is published with open access at Springerlink.com

\begin{abstract}
Experimental electron-impact ionization cross sections of one of the simplest carboxylic acids, formic acid, are presented. The molecular target was studied in two independent experimental setups applying different methods: the total ion collection and the electron-impact mass spectrometry methods. Experimental data were taken at incident electron energies ranging from ionization threshold $[11.31 \mathrm{eV}$; J.C. Traeger, Int. J. Mass Spectrom. Ion Process. 66, 271 (1985)] to $76 \mathrm{eV}$ using a total cross section of $4.0 \AA^{2}$ at $70 \mathrm{eV}$ for the total ion collection method. In the spectrometric method the four main cations $\mathrm{COH}^{+}$, $\mathrm{HCOOH}^{+}, \mathrm{COOH}^{+}$and $\mathrm{CO}^{+}$are found to contribute to about $97 \%$ of the total ionization cross section.
\end{abstract}

\section{Introduction}

Collision of low energy electrons with organic molecules has been the subject of many studies over the last few decades. However, comprehensive and reliable sets of cross section are still very much needed as input data for physico-chemical models [1].

Formic acid, $\mathrm{HCOOH}$, is the simplest carboxylic acid that can be found in many biological systems and is one of the simplest building blocks of biomolecules [2]. It is worth noting that recent theoretical first-principle molecular dynamics studies of Kohanoff and Artacho [3] predicted production of formic acid during radiolysis of water in low-energy carbon projectile bombardment. Additionally, formic acid (FA) plays an important role in the atmosphere, where it has an effect on precipitation chemistry and acidity [4]. Apart of its presence in the Earth's atmosphere, FA has also been detected in the interstellar medium (ISM): in dark clouds [5], in the Galactic center (GC) [6], in the cometary coma of Hale-Bopp comet [7], in interstellar ices [8] and in chondritic meteorites [9]. All these discoveries have made the research of the interaction of FA with free electrons important from the point of view of atmospheric physics, astrophysics, astrobiology and post-irradiation chemistry [10].

Among reports on electron interaction with FA one finds many experimental studies of dissociative electron attachment cross sections [11-14], differential and momentum transfer cross sections $[15,16]$ and absolute differential

\footnotetext{
* Contribution to the Topical Issue "Low Energy Positron and Electron Interactions", edited by James Sullivan, Ron White, Michael Bromley, Ilya Fabrikant and David Cassidy.

${ }^{a}$ e-mail: mateusz.zawadzki@jh-inst.cas.cz
}

elastic and vibrational excitation cross sections [17]. In order to obtain ionization appearance-potientials, fragmentation of FA monomer by electron impact was also studied by Mariner and Bleakney [18]. There were also several reports on determination of ionization appearanceenergies using photoionization mass spectrometry method [19-24].

Furthermore, there are a number of theoretical studies on FA, including studies on nuclear dynamics of low-energy electron attachment [25], dissociative electron attachment using Feshbach resonance theory [26] as well as studies of resonant behavior using the R-matrix theory [27], calculations of momentum transfer and angular differential cross sections using fixed-nuclei complex Kohn variational calculations [28] or the Schwinger multichannel method with pseudopotentials [29]. Further theoretical studies of differential cross sections for the electron impact ionization of the outer valence orbitals of FA, applying the molecular three-body distorted wave (M3DW) approximation [30], in the determination of triple-differential cross sections for the electron-impact ionization carried out by the multicenter distorted-wave method [31], have been made.

From the above perspective it is surprising that for this important molecule the only available experimental data on absolute electron ionization cross section are those of Pilling et al. [32]. Pilling and coworkers studied ionization and dissociation of cometary gaseous organic molecules by solar wind particles. However, their absolute ionization and dissociation cross sections for FA interacting with electrons were measured employing only a few electron energies $(70 \mathrm{eV}, 0.5 \mathrm{keV}, 1 \mathrm{keV}$ and $2 \mathrm{keV})$, not covering the gap for low energy electron impact closer to threshold. The method applied by Pilling et al. [32], for 


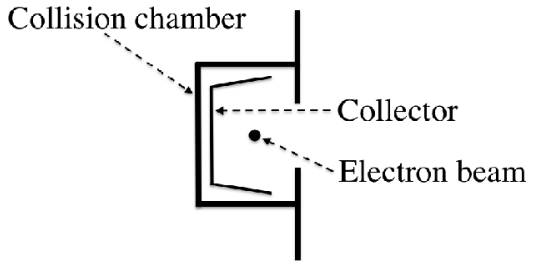

Fig. 1. The scheme of the U-shaped ion collector. The orientation of electron beam is perpendicular to the paper plane. For further details see references [36,37].

the electron impact experiment, consisted of a continuous electron beam crossed perpendicularly by the gaseous formic acid target jet. Ionic fragments resulting from the collisions were analysed by means of a time-of-flight mass spectrometer, employing a pulsed extraction system.

Electron impact ionization cross sections for FA were calculated using different theoretical approaches. Vinodkumar et al. [33] presented theoretical calculations of the total ionization cross sections for electron impact on FA using a multicenter group additivity rule for impact energies of 15-2000 eV. The same group performed calculations employing an Improved Complex Scattering Potential-ionization contribution (ICSP-ic) to obtain ionization cross sections from total inelastic cross sections for energies ranging from $10 \mathrm{eV}$ to $5000 \mathrm{eV}$ incorporating the binary-encounter-Bethe model (BEB) in the same energy range [34]. Additionally, cross sections for single electronimpact ionization of FA were calculated using the BEB model in the energy range from the ionization threshold of $11.31 \mathrm{eV}$ [19] up to $4000 \mathrm{eV}$ [35]. There the outer valence Green function (ROVGF) calculations of correlated electron affinities and ionization potentials were performed, providing different input parameters for the two BEB model calculations. For this reason, one can observe the difference between the BEB model calculations used by Możejko [35] and Vinodkumar et al. [34].

The purpose of the present work is to provide the cross sections for electron-impact ionization of FA in an incident electron energy range, which has not been measured experimentally for comparison with theoretical models.

\section{Experimental methods}

We performed measurements of electron-impact ionization cross sections using two independent experimental approaches. These were the total ion collection method and the variable energy time-of-flight (TOF) spectrometry method. The former method measures total ionization cross-sections whereas the latter method provides relative partial cross sections. Both these methods will be described in the following sections.

\subsection{The total ionization cross sections}

For the total ionization cross section measurement we adapted an existing apparatus, which was used for obtaining absolute dissociative electron attachment cross sections [36]. The setup was used in the total ion collection mode as described previously by Chachereau et al. [37]. Electrons with well defined energy were emitted from a filament and passed through the trochoidal monochromator, and were focused inside a collision chamber. The controlling of electron current was ensured by electron collection on a Faraday cup, which was placed after the collision chamber. The U-shaped ion collector, in the original experiment used as a negative ion repeller, was held at a slightly negative potential (reaching the maximum ion collection signal) to ensure collection of positive ions and repulsion of negative fragments. The U-shaped ion collector, which surrounded the ionization region, is shown in the schematic picture (Fig. 1).

The electron monochromator with ionization cell was housed inside a vacuum chamber with a typical pressure of around $10^{-7}$ mbar. With the target gas flowing, the pressure in the collision region was typically in the range 3 to $6 \times 10^{-4}$ mbar, measured using a MKS baratron model 670 capacitance manometer. The electron beam current was typically $8-12 \mathrm{nA}$ and the energy resolution of the electron beam was $200 \mathrm{meV}$. The electron interaction path length was $20 \mathrm{~mm}$, which was taken to be the same as the length of the ion collector in the reaction chamber. During the course of experiment, the ion currents were recorded as a function of the incident electron energy. The ion current at the collector was recorded using a Keithley model 617 programmable electrometer, which then was connected through a Keithley model 199 system DMM scanner to a computer unit. The ion current recorded during the course of experiment was in the pA range.

The absolute TICS, $\sigma(E)$, can be obtained as

$$
\sigma(E)=\frac{k_{\mathrm{B}} T}{p l} \frac{I_{\text {ion }}}{I_{\text {electron }}},
$$

where $I_{\text {ion }}$ stands for the ion current and $I_{\text {electron }}$ for the electron current, $k_{\mathrm{B}}$ is the Boltzmann constant, $p$ and $T$ are the pressure and temperature of the sample gas, respectively, $l$ is the length of the interaction path.

The stability of the experiment allowed us to produce data with excellent reproducibility. Before and after measurement of the target gas, several measurements of well known ionization curves for $\mathrm{CO}_{2}$ and $\mathrm{SF}_{6}$ were performed. Additionally, a background measurement was regularly taken to ensure no other impurities were present in the chamber.

The sample of $\mathrm{HCOOH}$ used was from Sigma-Aldrich and had a stated purity of $95 \%$. Before measurement FA was purified with repeated freeze-pump-thaw cycles at the liquid- $\mathrm{N}_{2}$ temperature to remove dissolved air and other volatile impurities. For reference measurements, commercially supplied samples of $\mathrm{CO}_{2}$ and $\mathrm{SF}_{6}$ were used with purities of $99.80 \%$ and $99.75 \%$, respectively.

The uncertainties in total ionization cross section may arise from several contributions, both statistical and systematic. When calculating the overall error for measured data we included an estimated $\pm 5 \%$ uncertainty associated with ion collection efficiency, a $\pm 2 \%$ uncertainty in the electron beam current measurement, a $\pm 0.5 \%$ uncertainty in the calibration of the electrometer used for the electron beam current measurement, $a \pm 2 \%$ uncertainty in 
Table 1. Total cross section for electron impact ionization of formic acid and uncertainty limits in units of $10^{-20} \mathrm{~m}^{2}$.

\begin{tabular}{|c|c|c|c|c|c|}
\hline Electron energy $[\mathrm{eV}]$ & TICS & Uncertainty & Electron energy $[\mathrm{eV}]$ & TICS & Uncertainty \\
\hline 10.67 & 0.000 & \pm 0.000 & 44.08 & 3.38 & \pm 0.51 \\
\hline 11.63 & 0.015 & \pm 0.002 & 45.00 & 3.43 & \pm 0.51 \\
\hline 12.56 & 0.051 & \pm 0.008 & 45.93 & 3.48 & \pm 0.52 \\
\hline 13.43 & 0.104 & \pm 0.016 & 46.86 & 3.52 & \pm 0.53 \\
\hline 14.41 & 0.171 & \pm 0.026 & 47.78 & 3.56 & \pm 0.53 \\
\hline 15.34 & 0.251 & \pm 0.038 & 48.71 & 3.60 & \pm 0.54 \\
\hline 16.27 & 0.348 & \pm 0.052 & 49.64 & 3.64 & \pm 0.55 \\
\hline 17.18 & 0.448 & \pm 0.067 & 50.57 & 3.67 & \pm 0.55 \\
\hline 18.12 & 0.562 & \pm 0.084 & 51.49 & 3.70 & \pm 0.56 \\
\hline 19.05 & 0.677 & \pm 0.102 & 52.42 & 3.74 & \pm 0.56 \\
\hline 19.96 & 0.799 & \pm 0.120 & 53.35 & 3.76 & \pm 0.56 \\
\hline 20.90 & 0.918 & \pm 0.138 & 54.27 & 3.79 & \pm 0.57 \\
\hline 21.83 & 1.05 & \pm 0.16 & 55.20 & 3.81 & \pm 0.57 \\
\hline 22.76 & 1.17 & \pm 0.18 & 56.13 & 3.84 & \pm 0.58 \\
\hline 23.68 & 1.30 & \pm 0.20 & 57.05 & 3.85 & \pm 0.58 \\
\hline 24.61 & 1.43 & \pm 0.21 & 57.98 & 3.87 & \pm 0.58 \\
\hline 25.53 & 1.56 & \pm 0.23 & 58.91 & 3.88 & \pm 0.58 \\
\hline 26.46 & 1.68 & \pm 0.25 & 59.83 & 3.89 & \pm 0.58 \\
\hline 27.39 & 1.80 & \pm 0.27 & 60.76 & 3.91 & \pm 0.59 \\
\hline 28.32 & 1.92 & \pm 0.29 & 61.69 & 3.93 & \pm 0.59 \\
\hline 29.24 & 2.04 & \pm 0.31 & 62.61 & 3.95 & \pm 0.59 \\
\hline 30.17 & 2.15 & \pm 0.32 & 63.54 & 3.96 & \pm 0.59 \\
\hline 31.10 & 2.26 & \pm 0.34 & 64.47 & 3.97 & \pm 0.60 \\
\hline 32.03 & 2.36 & \pm 0.35 & 65.40 & 3.99 & \pm 0.60 \\
\hline 32.95 & 2.46 & \pm 0.37 & 66.32 & 4.01 & \pm 0.60 \\
\hline 33.88 & 2.56 & \pm 0.38 & 67.25 & 4.01 & \pm 0.60 \\
\hline 34.81 & 2.65 & \pm 0.40 & 68.18 & 4.02 & \pm 0.60 \\
\hline 35.73 & 2.75 & \pm 0.41 & 69.10 & 4.02 & \pm 0.60 \\
\hline 36.66 & 2.83 & \pm 0.42 & 70.03 & 4.01 & \pm 0.60 \\
\hline 37.58 & 2.91 & \pm 0.44 & 70.95 & 4.01 & \pm 0.60 \\
\hline 38.51 & 2.99 & \pm 0.45 & 71.89 & 4.01 & \pm 0.60 \\
\hline 39.44 & 3.07 & \pm 0.46 & 72.81 & 4.00 & \pm 0.60 \\
\hline 40.37 & 3.14 & \pm 0.47 & 73.74 & 3.99 & \pm 0.60 \\
\hline 41.30 & 3.21 & \pm 0.48 & 74.66 & 3.99 & \pm 0.60 \\
\hline 42.22 & 3.27 & \pm 0.49 & 75.59 & 3.98 & \pm 0.60 \\
\hline 43.15 & 3.32 & \pm 0.50 & & & \\
\hline
\end{tabular}

the ion current measurement, a $\pm 1 \%$ uncertainty in the calibration of the electrometer used for the ion current measurement, a $\pm 2 \%$ uncertainty of the DMM scannerdigital multimeter and its calibration $( \pm 1 \%)$, a $\pm 2 \%$ uncertainty in the ion extraction length, a $\pm 2 \%$ statistical uncertainty and a $\pm 1 \%$ calibration uncertainty in the pressure measurement. The above errors were added in quadrature to obtain the final total error. All the corresponding total errors are presented in Table 1.

\subsection{The relative partial ionization cross sections}

Present measurements of the relative partial ionization cross sections were obtained using a part of a complex CLUster Beam (CLUB) apparatus [38]. In this work, only the description of the section directly related to the performed experiment is provided; more detailed information can be found elsewhere [39].
The positive mass spectra of FA were measured using a RTOF MS: a double stage reflectron system with WilleyMcLaren ion extraction. The sample gas was introduced into the reaction chamber at pressures between 2 and $5 \times 10^{-7} \mathrm{mbar}$. The interaction/extraction region included two parallel plates $(45 \mathrm{~mm} \times 72 \mathrm{~mm}$ each) separated by $14 \mathrm{~mm}$, where one (extraction plate) had an exit slit $(3 \mathrm{~mm} \times 10 \mathrm{~mm})$, through which ions entered the time-offlight region. A short $300 \mathrm{~ns}$ pulse of electrons was sent through the interaction region while the ion repeller and extraction plate were on the potential of the chamber. Subsequently, a $2 \mu$ s pulse was applied to the repeller and the extraction plate with an amplitude of $-1.5 \mathrm{kV}$ and $+1.5 \mathrm{kV}$, respectively. The experiment was repeated at a rate of $5 \mathrm{kHz}$. Ions were extracted perpendicularly to the beam and accelerated to the final kinetic energy of $6 \mathrm{kV}$. After passing the effective flight path of $0.95 \mathrm{~m}$ ions were detected onto a $4 \mathrm{~cm}$ in diameter Photonics MCP detector in a Chevron configuration. 


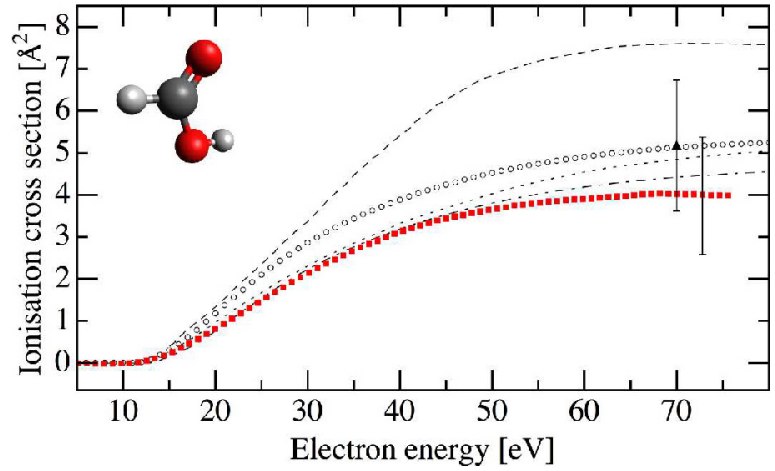

Fig. 2. The total ionization cross section of formic acid molecules. Experimental data: (ם) present results, (ム) Pilling et al. [32]; theoretical data: $(-\cdot-)$ using BEB method and $(\cdots)$ using ICSP-ic method by Vinodkumar et al. [34], (o) Możejko [35], (- - -) Vinodkumar et al. [33]. In the top left corner a schematic of the FA molecule.

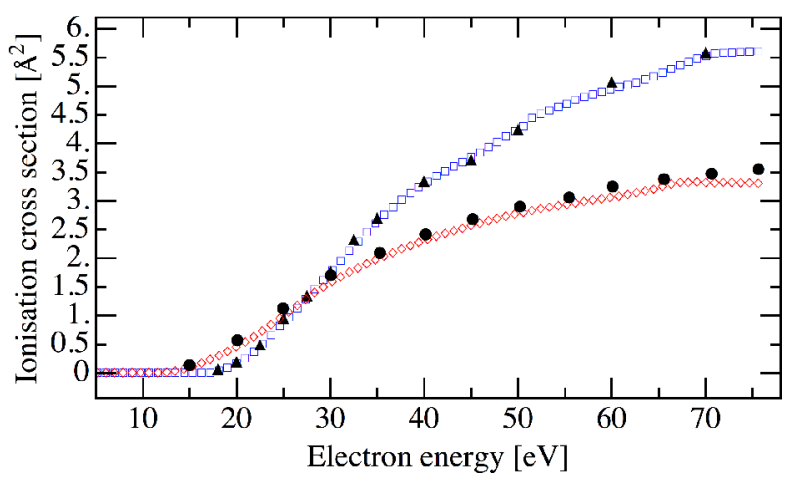

Fig. 3. Cross sections for electron-impact ionization of $\mathrm{CO}_{2}$ : $(\diamond)$ this work, $(\bullet)$ literature data from Straub et al. [42]; and $\mathrm{SF}_{6}$ : $(\square)$ this work, $(\boldsymbol{\Delta})$ literature data from Rejoub et al. [43].

The ion signal was measured as a function of two parameters: mass/charge ratio and electron energy. Specific mass spectra and electron energy dependent ion yields were then extracted from the data obtained in the course of the experiment.

\section{Results and discussion}

\subsection{Total ionization cross sections}

Total electron-impact ionization cross section of FA molecules using the total ion collection technique was measured for low to intermediate energies. The results are presented in Figure 2 and are listed in Table 1. The figure also shows theoretical predictions available in literature [33-35]. Additionally, within measured energy range we are able to include an experimental point of Pilling et al. [32] at $70 \mathrm{eV}$.

With the assumption that all excess energy is directed into ionization channel, the BEB theory provides an upper-bound TICS. However, as this is not the only available process during the electron impact, the BEB model usually overestimates the ionization cross section [40,41].
The BEB calculations from Vinodkumar et al. [34] are higher than present experimental data at $70 \mathrm{eV}$ by about $10 \%$, whereas the same model with different input parameters used by Możejko [35] provided cross sections higher by $27 \%$. Also, different methods employed by Vinodkumar et al. $[33,34]$ predict considerably greater values of the TICS. At $70 \mathrm{eV}$ the Spherical Complex Optical Potential formalism (SCOP) overestimates the TICS by $89 \%$, whereas the Improved Complex Scattering Potential-ionization contribution (ICSP-ic) method is greater by $21 \%$.

For incident electron energies in the range of $<40 \mathrm{eV}$, the present measurements are in best agreement with the theoretical BEB and ICSP-ic models calculated by Vinodkumar et al. [34].

The only available experimental data for total electron impact ionization cross section of FA were provided by Pilling et al. [32]. For energies $\geq 0.5 \mathrm{keV}$ their relative intensities were measured experimentally, whereas for the $70 \mathrm{eV}$ electron-impact cross section they used a scaling based on the partial ion yield from the NIST data base. For this incident energy Pilling and coworkers indicate a total ionization cross section of $5.18 \AA^{2}$. However, the estimated error of this measurement is quite large at around $30 \%$. Their data point nevertheless agrees within its error limits with the present work.

As a test of our experiment calibration, our experimental data for $\mathrm{CO}_{2}$ and $\mathrm{SF}_{6}$ were compared with recommended sets of electron-impact ionization cross sections of Straub et al. [42] and Rejoub et al. [43], which are well established. Our recently measured TICS for these two targets show very good agreement with them over all the all energy range under study as is shown in Figure 3.

\subsection{TOF relative partial and total ionization cross sections}

The mass spectrum of formic acid cations was recorded at different electron energies, ranging from $11.31 \mathrm{eV}$ to $76 \mathrm{eV}$. The mass spectrum at an electron impact energy of $70 \mathrm{eV}$, together with assignments of the most dominant mass peaks, is shown in Figure 4. The corresponding relative abundances of the cation yields for main fragments are displayed in Table 2. At this incident electron energy of $70 \mathrm{eV}$, the most intense peak is of mass $29 \mathrm{amu}$, which corresponds to the $\mathrm{COH}^{+}$fragment i.e., break of the $\mathrm{C}-$ $\mathrm{OH}$ bond in FA. On the whole, one can distinguish four prominent cations from the ionization of FA molecules: $\mathrm{COH}^{+}, \mathrm{HCOOH}^{+}, \mathrm{COOH}^{+}, \mathrm{CO}^{+}$. These four dominant fragments contribute together to about $97 \%$ of the total cross section. Figure 5 depicts the total ionization cross section of formic acid and relative partial ionization cross sections of seven the most abundant cations. For completness, the partial ionization cross sections for three less prominent cations $\left(\mathrm{CO}_{2}{ }^{+}, \mathrm{OH}^{+}, \mathrm{O}^{+}\right)$were added and presented as one set of data. The total ionization cross section was considered as a sum of the partial yields for the main measured fragments. One could also observe ions with relative intensity of $<0.5 \%$ produced from $\mathrm{HCOOH}$ by electron impact. Due to their small contribution to TICS signal they were omitted in the total ion yield analysis. 


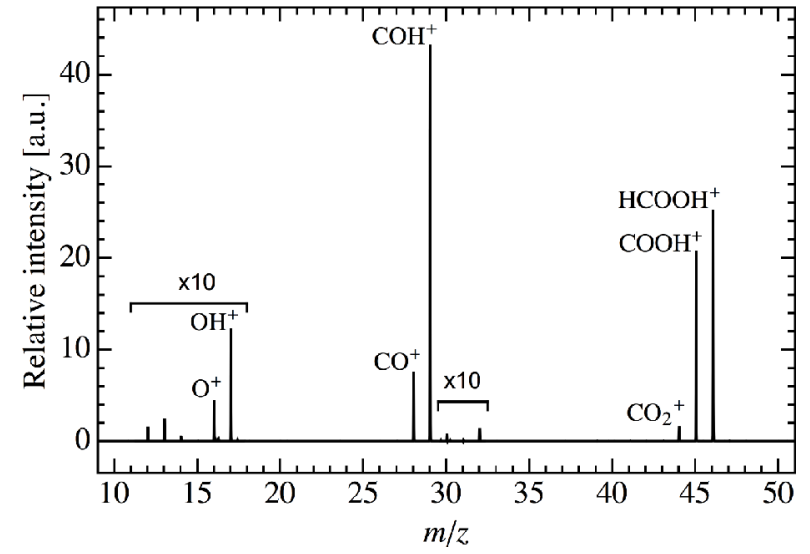

Fig. 4. The mass spectrum of cations of formic acid molecules recorded at an electron energy of $70 \mathrm{eV}$, with the relative assignment of main cationic fragments. Four dominant fragments, $\mathrm{COH}^{+}, \mathrm{HCOOH}^{+}, \mathrm{COOH}^{+}, \mathrm{CO}^{+}$, contribute together to about $97 \%$ of the total cross section.

Table 2. Relative abundances and assignments of main cations of formic acid molecules at $70 \mathrm{eV}$.

\begin{tabular}{lll}
\hline$m / z$ & Cation assignment & Relative abundance at $70 \mathrm{eV}$ \\
\hline 46 & $\mathrm{HCOOH}^{+}$ & $25.2 \%$ \\
45 & $\mathrm{COOH}^{+}$ & $20.8 \%$ \\
44 & $\mathrm{CO}_{2}^{+}$ & $1.6 \%$ \\
29 & $\mathrm{COH}^{+}$ & $43.1 \%$ \\
28 & $\mathrm{CO}^{+}$ & $7.6 \%$ \\
17 & $\mathrm{OH}^{+}$ & $1.2 \%$ \\
16 & $\mathrm{O}^{+}$ & $0.4 \%$ \\
\hline
\end{tabular}

For comparison, the total ionization cross section from the total ion collection experiment of formic acid molecules was also presented in Figure 5. Scaling of the sum of relative partial ionization cross sections to the total ion collection ionization signal in the range of incident energies below $40 \mathrm{eV}$ allows one to obtain the form of the partial relative cross-sections in the entire range. For energies $>40 \mathrm{eV}$ one can see the increasing difference between these two ionic signals. At $70 \mathrm{eV}$ the difference reaches $20 \%$. The reason for this can be due to several factors.

The RTOF mass spectrometer is primary used in molecular beam experiment and is placed perpendicular to the molecular beam axis [38]. To compensate the high kinetic energies in the molecular beam, the electron beam is tilted slightly off the central axis of the TOF extraction region (upstream the molecular beam). When analyzing static gas as in the present experiment this compensation of the kinetic energy of the molecules is not needed and using an off-axis electron beam setting results in the partial discrimination of the fragment ions.

Moreover, an underestimation of the ionization crosssection at higher electron energies can be a result of discrimination against fast ions with high kinetic energy release or doubly charged ions dissociating by Coulombic repulsion, as reported by Bull et al. [44]. As these processes are more probable with increasing electron energy and therefore also explain the observed difference between

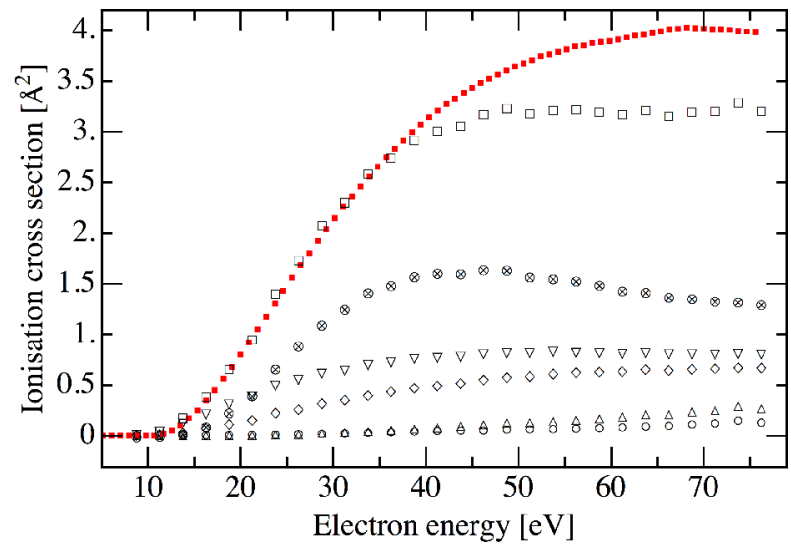

Fig. 5. (a) ( $\square$ ) The total ionization cross section of formic acid molecules derived from the partial ionization cross sections of the $(\otimes) \mathrm{COH}^{+},(\nabla) \mathrm{HCOOH}^{+},(\diamond) \mathrm{COOH}^{+},(\triangle) \mathrm{CO}^{+}$ and (o) $\mathrm{CO}_{2}{ }^{+}+\mathrm{OH}^{+}+\mathrm{O}^{+}$cations obtained at $70 \mathrm{eV}$; (b) the total ionization cross section $(\mathbf{\square})$ from the total ion collection experiment (Sect. 2.1).

total and partial cross section measurements. Other possible contributions to the observed difference could be the loss of some fragments in the reflectron mirror. This is common as the voltage of the last grid of the mirror required for optimal compensation for the initial kinetic energies of the fragments is often lower than required for total ion reflection. This way low mass fragments or high kinetic energy fragments may be lost and not detected, depending on the setting of the last grid voltage. Both types of fragments are primary formed at higher energies of incident electrons and this could explain the observed difference.

\section{Conclusions}

Absolute total cross sections for electron impact ionization of formic acid molecule, $\mathrm{HCOOH}$, have been measured at impact energies from threshold of $11.31 \mathrm{eV}$ to $76 \mathrm{eV}$. Two independent complementary instruments were used. First, are based on a total ion collection technique and second are using the electron impact mass spectroscopy method. The latter provided evidence for the production of dominating cations $\mathrm{COH}^{+}, \mathrm{HCOOH}^{+}, \mathrm{COOH}^{+}, \mathrm{CO}^{+}$, $\mathrm{CO}_{2}{ }^{+}, \mathrm{OH}^{+}$and $\mathrm{O}^{+}$from electron-impact ionization of $\mathrm{HCOOH}$.

This work provides very useful experimental data for an important, simple organic molecule in the incident electron energy range that had not been covered experimentally before. The previously published experimental point for an electron-impact ionization cross section of $\mathrm{HCOOH}$ (Pilling et al. [32]) is somewhat higher than the present results, but agrees within experimental errors. Our total cross sections for $\mathrm{CO}_{2}$ and $\mathrm{SF}_{6}$, used as a reference, are found to be in excellent agreement with the previous measurements available in literature, and serves as a good check of performance of the present experiment.

Additionally, when the present experimental data were compared to available theoretical calculations, it was 
shown that the theoretical models tend to overestimate the total electron-impact ionization cross section for formic acid.

This work has been supported by the Czech Science Foundation Grants 16-10995Y and 17-04844S. Author would like to thank J. Fedor, J. Kočišek, M. Fárník and M.A. Khakoo for fruitful discussions and valuable remarks.

Open Access This is an open access article distributed under the terms of the Creative Commons Attribution License (http://creativecommons.org/licenses/by/4.0), which permits unrestricted use, distribution, and reproduction in any medium, provided the original work is properly cited.

\section{References}

1. S.D. Rodgers, S.B. Charnley, W.F. Huebner, D.C. Boice, in Comets II, edited by M.C. Festou, H.U. Keller, H.A. Weaver (University of Arizona Press, Tucson, 2004), Vol. 745

2. G.A. Kumar, Y. Pan, C.J. Smallwood, M.A. McAllister, J. Comput. Chem. 19, 1345 (1998)

3. J. Kohanoff, E. Artacho, PLoS ONE 12, 3 (2017)

4. D.B. Millet, M. Baasandorj, D.K. Farmer, J.A. Thornton, K. Baumann, P. Brophy, S. Chaliyakunnel, J.A. de Gouw, M. Graus, L. Hu, A. Koss, B.H. Lee, F.D. Lopez-Hilfiker, J.A. Neuman, F. Paulot, J. Peischl, I.B. Pollack, T.B. Ryerson, C. Warneke, B.J. Williams, J. Xu, Atmos. Chem. Phys. 15, 6283 (2015)

5. W.M. Irvine, P. Friberg, N. Kaifu, Y. Kitamura, K. Kawaguchi, Astrophys. J. 342, 871 (1989)

6. M.A. Requena-Torres, J. Martín-Pintado, A. RodríguezFranco, S. Martín, N.J. Rodríguez-Fernández, P. de Vicente, Astron. Astrophys. 455, 971 (2006)

7. D. Bockelée-Morvan, D.C. Lis, J.E. Wink, D. Despois, J. Crovisier, R. Bachiller, D.J. Benford, N. Biver, P. Colom, J.K. Davies, E. Gerard, B. Germain, M. Houde, D. Mehringer, R. Moreno, G. Paubert, T.G. Phillips, H. Rauer, Astron. Astrophys. 353, 1101 (2000)

8. J.V. Keane, A.G.G.M. Tielens, A.C.A. Boogert, W.A. Schutte, D.C.B. Whittet, Astron. Astrophys. 376, 254 (2001)

9. J.F. Briscoe, C.B. Moore, Meteoritics 28, 330 (1993)

10. S. Cuadrado, J.R. Goicoechea, O. Roncero, A. Aguado, B. Tercero, J. Cernicharo, Astron. Astrophys. 596, L1 (2016)

11. A. Pelc, W. Sailer, P. Scheier, N.J. Mason, T.D. Märk, Eur. Phys. J. D 20, 441 (2002)

12. A. Pelc, W. Sailer, P. Scheier, T.D. Märk, Vaccum 78, 631 (2005)

13. R. Janečková, D. Kubala, O. May, J. Fedor, M. Allan, Phys. Rev. Lett. 111, 213201 (2013)

14. V.S. Prabhudesai, D. Nandi, A.H. Kelkar, R. Parajuli, E. Krishnakumar, Chem. Phys. Lett. 405, 172 (2005)
15. F.A. Gianturco, R.R. Lucchese, Eur. Phys. J. D 39, 399 (2006)

16. V. Vizcaino, M. Jelisavcic, J.P. Sullivan, S.J. Buckman, New J. Phys. 8, 85 (2006)

17. M. Allan, J. Phys. B: At. Mol. Opt. Phys. 39, 2939 (2006)

18. T. Mariner, W. Bleakney, Phys. Rev. 72, 792 (1947)

19. J.C. Traeger, Int. J. Mass Spectrom. Ion Process. 66, 271 (1985)

20. K. Watanabe, J. Chem. Phys. 26, 542 (1957)

21. F.I. Vilesov, Dokl. Phys. Chem. 132, 521 (1960)

22. C.S. Matthews, P. Warneck, J. Chem. Phys. 51, 854 (1969)

23. D.J. Knowles, A.J.C. Nicholson, J. Chem. Phys. 60, 1180 (1974)

24. P. Warneck, Z. Naturforsch. A 29, 350 (1974)

25. T.N. Rescigno, C.S. Trevisan, A.E. Orel, Phys. Rev. Lett. 96, 213201 (2006)

26. G.A. Gallup, Phys. Rev. A 88, 052705 (2013)

27. G.A. Gallup, P.D. Burrow, I.I. Fabrikant, Phys. Rev. A 79, 042701 (2009)

28. C.S. Trevisan, A.E. Orel, T.N. Rescigno, Phys. Rev. A 74, 042716 (2006)

29. M.H.F. Bettega, Phys. Rev. A. 74, 054701 (2006)

30. C.J. Colyer, M.A. Stevenson, O. Al-Hagan, D.H. Madison, C.G. Ning, B. Lohmann, J. Phys. B: At. Mol. Opt. Phys. 42, 235207 (2009)

31. X. Li, M. Gong, L. Liu, Y. Wu, J. Wang, Y. Qu, X. Chen, Phys. Rev. A 95, 012703 (2017)

32. S. Pilling, A.C.F. Santos, W. Wolff, M.M. Sant'Anna, A.L.F. Barros, G.G.B. de Souza, N.V. de Castro Faria, H.M. Boechat-Roberty, Mon. Not. R. Astron. Soc. 372, 1379 (2006)

33. M. Vinodkumar, K.N. Joshipura, C. Limbachiya, N. Mason, Phys. Rev. A 74, 022721 (2006)

34. M. Vinodkumar, H. Bhutadia, B. Antony, N. Mason, Phys. Rev. A 84, 052701 (2011)

35. P. Możejko, Eur. Phys. J. Special Topics 144, 233 (2007)

36. O. May, J. Fedor, B.C. Ibănescu, M. Allan, Phys. Rev. A 77, 040701(R) (2008)

37. A. Chachereau, J. Fedor, R Janečková, J. Kočišek, M. Rabie, C.M. Franck, J. Phys. D: Appl. Phys. 49, 375201 (2016)

38. M. Fárník, Molecular dynamics in free clusters and nanoparticles studied in molecular beams (ICT Prague Press, Prague, 2011)

39. J. Kočišek, J. Lengyel, M. Fárník, J. Chem. Phys. 138, $124306(2013)$

40. J.N. Bull, P.W. Harland, C. Vallance, J. Phys. Chem. A 116, 767 (2012)

41. J.N. Bull, M. Bart, C. Vallance, P.W. Harland, Phys. Rev. A 88, 062710 (2013)

42. H.C. Straub, B.G. Lindsay, K.A. Smith, R.F. Stebbings, J. Chem. Phys. 105, 10 (1996)

43. R. Rejoub, D.R. Sieglaff, B.G. Lindsay, R.F. Stebbings, J. Phys. B: At. Mol. Opt. Phys. 34, 1289 (2001)

44. J.N. Bull, J.W.L. Lee, C. Vallance, Phys. Chem. Chem. Phys. 16, 10743 (2014) 